\title{
Sequential Electro-Anatomical Mapping Methodology and Preliminary Results for Reentry Vulnerability Index Estimation
}

\author{
Michele Orini ${ }^{1,2}$, Ben Hanson ${ }^{1}$, Peter Taggart ${ }^{1}$, Fernando Campos $^{3}$, Aldo Rinaldi ${ }^{3}$, Jaswinder Gill ${ }^{3}$, \\ Martin Bishop ${ }^{3}$, Pier D Lambiase ${ }^{1}$ \\ ${ }^{1}$ University College London, London, United Kingdom \\ 2 Barts Heart Centre, St Bartholomew's Hospital, London, United Kingdom \\ ${ }^{3}$ King's College London, London, United Kingdom
}

\begin{abstract}
Ventricular tachycardia (VT) recurrence after catheter ablation remains frequent and improved ablation strategies are needed. The re-entry vulnerability index (RVI) is an activation-repolarization marker to localize critical sites for VT initiation. Its use is limited since current electro-anatomical mapping systems (EAMS) cannot provide global measurement of activation and repolarization times within a single beat. We carried out a simulation study to assess a simple method to measure RVI using data collected by sequential EAMS and we investigated the effect of background noise, $R T$ variability $\left(\sigma_{R T}\right)$ and ectopics on RVI estimation. The mean correlation coefficient between single ECG beats and a representative template is used as inclusion/exclusion criterion. Localization of the vulnerable region associated with $5 \%$ bottom RVI was accurate (sensitivity $80 \pm 8 \%$, specificity $>99 \pm 1 \%$ ) for moderate to large repolarization variability $\left(5 \leq \sigma_{R T} \leq 20\right.$ $m s)$ and moderate level of noise ( $S N R \geq 10 \mathrm{~dB})$ but it deteriorated for $\sigma_{R T} \geq 25 \mathrm{~ms}$ and $S N R \leq 5 \mathrm{~dB}$. Sensitivity remained high even when RVI estimates were only moderately accurate ( $c c>0.67 \pm 0.05, M A E<25 \pm 1 \mathrm{~ms})$. The number of ectopic beats did not affect the results. In the in-vivo case analyzed, the sites of low RVI and VT exit was close $(5.1 \mathrm{~mm})$.
\end{abstract}

\section{Introduction}

Ventricular tachycardia (VT) recurrence after catheter ablation remains frequent and improved ablation strategies are needed. The re-entry vulnerability index (RVI) is an activation-repolarization marker recently proposed [1] to localize critical sites for VT initiation and guide catheter ablation. This metric is based on the observation that a re-entry is usually initiated by the following events: A premature ventricular contraction with short coupling interval initiates an activation wavefront, which due to conduction restitution dynamics [2], proceeds with low conduction velocity; Propagation is blocked by either an anatomical (scar) or functional block; The wavefront travels around the line of block and attempts to re-enter; If the re-entrant wave-front finds tissue that has regained excitability ahead of it, it will conducts and the re-entrant circuit will initiate VT. On the other hand, if the tissue ahead of the reentrant wave-front is still refractory, the re-entrant wavefront will be blocked. This series of events established a relationship between activation-repolarization times at adjacent sites which is captured by the RVI metric. One of the limitation for RVI implementation is that current electroanatomical mapping systems (EAMS) cannot provide global measurement of activation and repolarization times within a single beat. Here we aim to (1) Assess a simple methodology to map RVI using sequential EAMS and (2) Provide preliminary results about the algorithm's ability to localize re-entry initiation sites.

\section{Methods}

An RVI map measuring the spatial distribution of the tissue susceptibility to re-entry can be estimated from repolarization and activation maps, $A T_{i}$ and $R T_{i}$, where $i=[1: N]$ are nodes of a detailed ventricular mesh. At each node $i, R V I_{i}$ can be calculated as the minimum difference between RT at site $i$ (proximal) and AT at neighbouring sites $j$ (distal) comprised within a given radius $[1,3]$ :

$$
R V I_{i}=\min _{j}\left(R T_{i}-A T_{j}\right)
$$

The lower this index, the higher the probability for a wave-front traveling along a line of block to find excitable tissue ahead of it and initiate a re-entry. A sequential mapping method assumes that the activation-repolarization sequence of consecutive beats is identical. In practice, this assumption is not verified as beat-to-beat variability of repolarization $[4,5]$, and, to lesser extent, activation exists. To measure $R T_{i}$ and $A T_{i}$ from different 

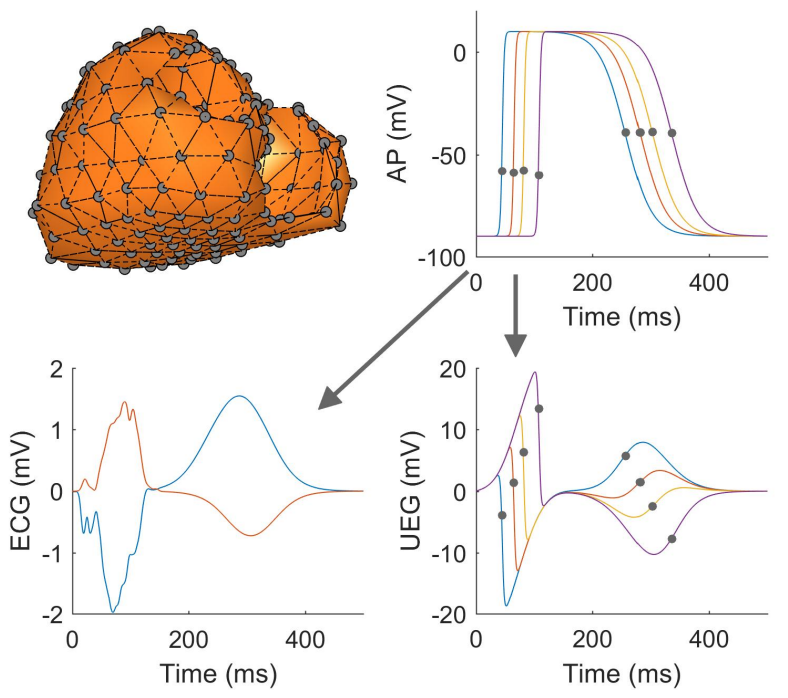

Figure 1. Simulation Framework. At each node of the ventricular mesh (top-left), an action potential is generated using analytical functions (top-right) using (2), while a unipolar electrogram (bottom-right) is generated using (3). Finally, independent ECG leads are estimated using the transformation matrix provided by ECG-Sim 4 .

beats, we assume that different beats characterized by similar activation-repolarization dynamics have similar surface ECGs. Therefore, the correlation between the ECG of a given beat and a representative ECG template can be used as inclusion criterion.

\section{Simulation Study}

Figure 1 describe the simulation study. A ventricular mesh of 257 nodes from ECG-Sim was used [6]. At each node, local action potentials (AP) were generated using the following analytical functions [7]:

$$
\begin{aligned}
V_{i}(t) & =A \frac{1}{1+\exp \left(-\beta_{A T}\left(t-\tau_{A T, i}\right)\right)} \\
& \left(1-\frac{1}{1+\exp \left(-\beta_{R T}\left(t-\tau_{R T, i}\right)\right)}\right)-V_{0}
\end{aligned}
$$

where $\beta_{A T}=0.600$ and $\beta_{R T}=0.045$ are constant that determine the steepness of the activation upslope and repolarization downslope, respectively, of all APs; $A=10$ and $V_{0}=-90 \mathrm{mV}$ are the amplitude and the resting potential of $V_{i}(t) ; \tau_{A T, i}$ and $\tau_{R T, i}$ are the AT and RT at cardiac site $x_{i}$, defined as the the moment of the steepest upslope and downslope in the AP, i.e. $\tau_{A T, i}=\arg \max \left(d V_{i}(t) / d t\right)$ and $\tau_{R T, i}=\arg \min \left(d V_{i}(t) / d t\right)$. According to a simple model, the UEG recorded at the same cardiac site is $[7,8]$ :

$$
U_{i}(t)=C\left(V_{\mathrm{R}}(t)-V_{i}(t)\right)
$$

\section{A. Activation Time}

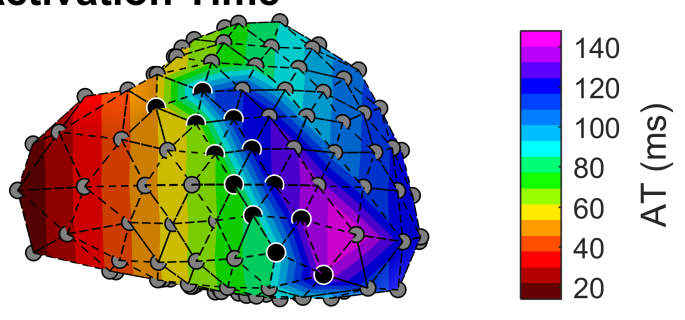

\section{B. Repolarization Time}

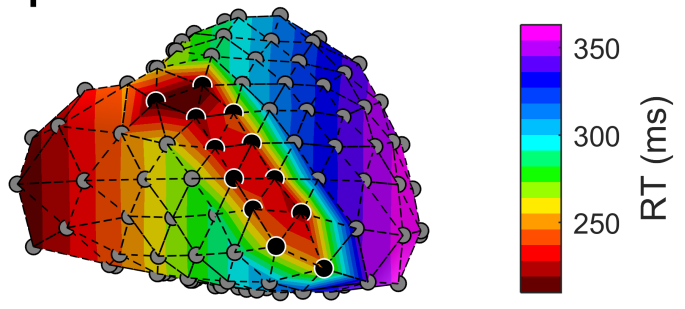

\section{RVI}

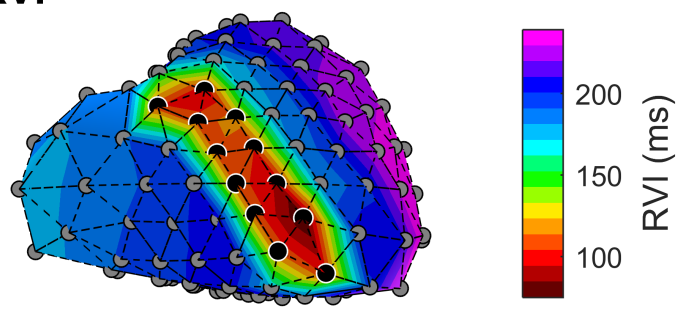

Figure 2. Activation, repolarization and RVI of the reference beat, showing a region of conduction slowing (top) adjacent to a region of early repolarization (middle), resulting in a small region of low RVI values (bottom, black circles).

where $C=0.25$ is a scaling factor that takes into account the difference between intra- and extracellular conductances [8], and $V_{\mathrm{R}}(t)$ is a position-independent potential that represents far field activities, defined as the arithmetic mean of all $V_{i}(t)$.

The surface ECG was measure using the formulation provided by ECG-Sim [6] as in previous studies [9]:

$$
E C G(t)=\mathbf{A} \times \mathbf{V}(\mathbf{t})
$$

An activation-repolarization sequence was simulated including a region of conduction slowing adjacent to a region of fast repolarization, resulting in a small region of low RVI (high susceptibility to re-entry) composed of 12 nodes (5\% of ventricular surface). The average RVI within the low RVI region was $103 \pm 11 \mathrm{~ms}$, while the average RVI in the normal tissue (outside the low-RVI region was) $206 \pm 16$. This is shown in Fig. 2.

Beat to beat variability was simulated by reproducing the 
basic activation-repolarization sequence for $n=1: 250$ beats and adding white Gaussian noise with variance $\sigma_{A T}^{2}$ and $\sigma_{R T}^{2}$ :

$$
\begin{aligned}
& \tau_{A T, i}(n)=\tau_{A T, i}+\sigma_{A T} \xi_{i}(n) \\
& \tau_{R T, i}(n)=\tau_{A T, i}+\sigma_{R T} \xi_{i}(n)
\end{aligned}
$$

where $\sigma_{A T}=1 \mathrm{~ms}$ and $\sigma_{R T}=\{5,10,25,25,30\} \mathrm{ms}$. Furthermore, background noise contaminating the recording was simulated by adding white Gaussian noise to the unipolar electrograms, with signal to noise ratio (SNR) equal to $5,10,15,20 \mathrm{~dB}$. Finally, $10 \%$ of beats were replaced by ectopics with different activation-repolarization sequence.

Sequential mapping was simulated by selecting 10 unipolar electrograms per beat as if data were recording using a decapolar catheter. The recordings were included in the analysis if the mean correlation coefficient between the ECG of the reference beat and the current beat was higher than 0.95 and discarded otherwise.

AT and RT were measured as the intervals between the pacing stimulus and the time of the minimum of the first derivative during the QRS complex and the maximum of the first derivative of the T-wave independently of its polarity, respectively. For each configuration of $\left\{\sigma_{A T}, \sigma_{R T}, S N R\right\}$, RVI estimation was repeated 50 times (50 realizations of random noise) and agreement between reference and estimated RVI was assessed using the mean absolute error (MAE) and correlation coefficient (cc). Delineation of low RVI region was assessed in terms of sensitivity/specificity.

\subsection{Electrophysiological studies}

Data from a procedure for catheter ablation of VT was recorded using CARTO3 and analyzed off-line. The origin of VT was localized and confirmed by pace matching.

\section{Results}

The accuracy of RVI estimation closely tracked the accuracy of RT (Fig. 3). Background noise had negligible effect for $S N R \geq 15 \mathrm{~dB}$ (Figure 3-left) and had a lower impact on the estimation error and correlation coefficient than RT variability. RT variability $\left(\sigma_{R T}\right)$ produced an almost linear increase to the estimation error and a substantial decrease of the correlation coefficient, which was lower than 0.8 for $\sigma_{R T} \geq 20 \mathrm{~ms}$ (Figure 3-right). As Fig. 3 (bottom panels) shows, the delineation of the low-RVI region was accurate (sensitivity $>80 \pm 8 \%$, specificity $>99 \pm 1 \%$ ) for moderate to large repolarization variability $\left(5 \sigma_{R T} \leq 20\right.$ ms) and moderate level of noise $(S N R \geq 10 \mathrm{~dB})$ but it deteriorated for $\sigma_{R T} \geq 25 \mathrm{~ms}$ and $S N R \leq 5 \mathrm{~dB}$. Sensitivity remained high even when RVI estimates were only
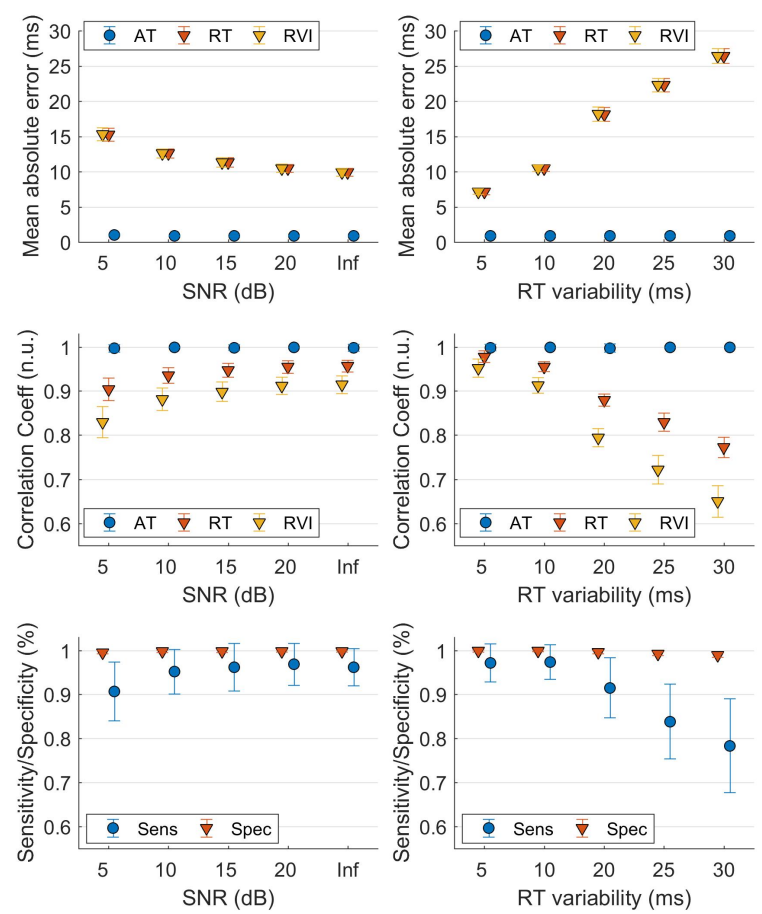

Figure 3. Results of the simulation study showing the effect of background signal noise (left) and repolarization variability (right), measured by $\sigma_{R T}$, on AT, RT and RVI estimation. Bottom panels show the accuracy in the delineation of the low-RVI region (sensitivity/specificity) as a function of SNR and $\sigma_{R T}$.

moderately accurate $(c c>0.67 \pm 0.05, M A E<25 \pm 1$ $\mathrm{ms})$. All ectopic beats were automatically discarded from the analysis due to low similarity between surface ECGs. Background noise did not increase the beats-rejection rate, meaning that it only mildly affects the morphology of the surface ECG. On the other hand, a marked increase of in the beats-rejection rate (and therefore in the duration of the procedure) was observed for $\sigma_{R T} \geq 20 \mathrm{~ms}$ (Fig. 4).

In in-vivo case analyzed, the low RVI region was adjacent to the VT exit site (distance $5.1 \mathrm{~mm}$, Fig. 5).

\section{Discussion}

RVI mapping is a promising technique to assess tissue susceptibility to arrhythmia and guide VT catheter ablation [1,3]. In this study, we assessed a simple method to measure RVI using data collected by sequential EAMS and we investigated the effect of background noise, RT variability and ectopics on RVI estimation. The similarity between surface ECGs of consecutive beat can be used to accept/reject beats underlying similar activationrepolarization sequences. This allows to measure RVI in the presence of ectopic beats and RT variability. Although 


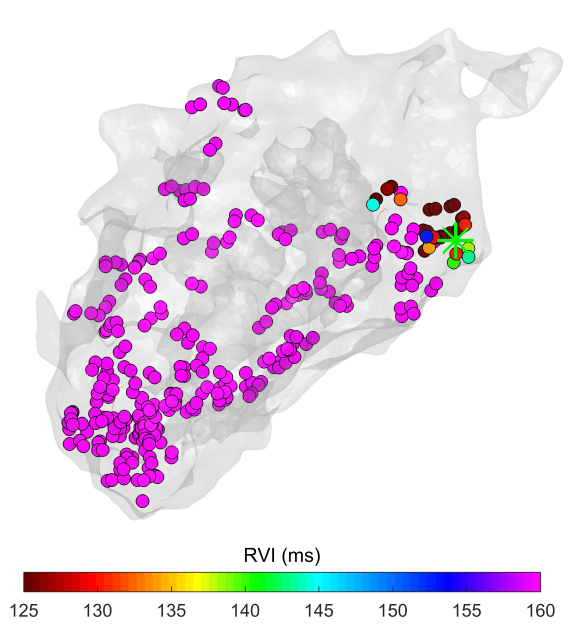

Figure 5. RVI LV map showing low-RVI region adjacent to VT exit site $($ green $*)$.
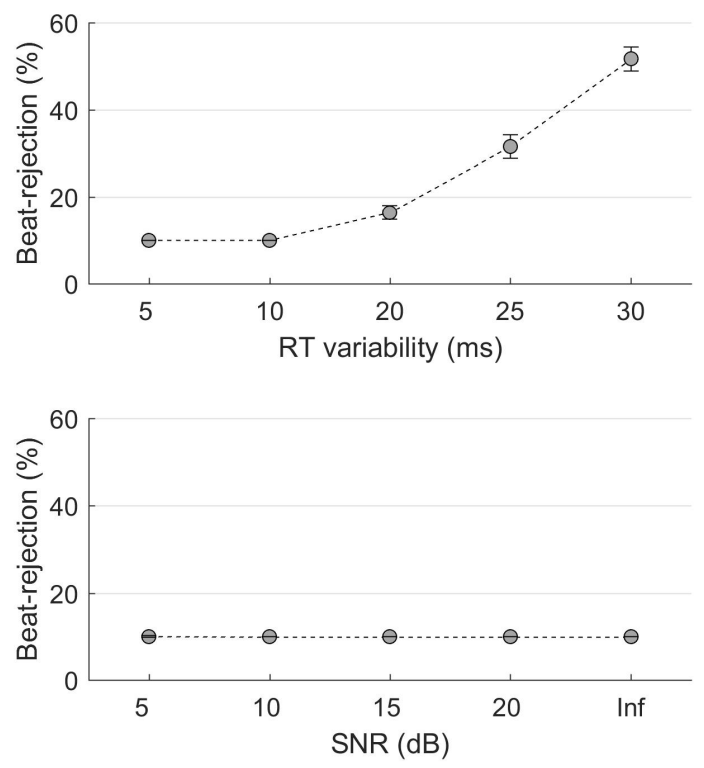

Figure 4. Beats-rejection rate (proportional to the procedure duration) as a function of SNR and RT variability. A beat is rejected if the mean correlation coefficient between its surface ECG and the reference ECG is $<0.95$.

RT variability can reduce the accuracy of RVI estimates, it only marginally affect the delineation of a low-RVI region. This simple analytical simulation suggests that RVI can be estimated from sequential EAMS and first results during in-vivo VT ablations are encouraging.

\section{References}

[1] Child N, Bishop MJ, Hanson B, Coronel R, Opthof T, Boukens BJ, Walton RD, Efimov IR, Bostock J, Hill Y, Rinaldi CA, Razavi R, Gill J, Taggart P. An activationrepolarization time metric to predict localized regions of high susceptibility to reentry. Heart Rhythm 2015;12(7):1644-53.

[2] Orini M, Taggart P, Srinivasan N, Hayward M, Lambiase PD. Interactions between activation and repolarization restitution properties in the intact human heart: In-vivo wholeheart data and mathematical description. PLoS ONE 2016; 11(9):e0161765.

[3] Martin CA, Orini M, Srinivasan NT, Bhar-Amato J, Honarbakhsh S, Chow AW, Lowe MD, Ben-Simon R, Elliott PM, Taggart P, Lambiase PD. Assessment of a conductionrepolarisation metric to predict Arrhythmogenesis in right ventricular disorders. International Journal of Cardiology 2018;ISSN 18741754.

[4] Orini M, Pueyo E, Laguna P, Bailon R. A Time-Varying Nonparametric Methodology for Assessing Changes in QT Variability Unrelated to Heart Rate Variability. IEEE Transactions on Biomedical Engineering 2018;65(7):1443-1451. ISSN 15582531.

[5] Orini M, Citi L, Hanson BMB, Taggart P, Lambiase PDP. Characterization of the Causal Interactions Between Depolarization and Repolarization Temporal Changes in Unipolar Electrograms. In Computers in Cardiology, volume 40. IEEE, 2013; 719-722.

[6] van Oosterom A. ECGSIM: an interactive tool for studying the genesis of QRST waveforms. Heart 2004;90(2):165-168. ISSN 0007-0769.

[7] Orini M, Taggart P, Lambiase PD. In vivo human sockmapping validation of a simple model that explains unipolar electrogram morphology in relation to conductionrepolarization dynamics. Journal of Cardiovascular Electrophysiology jul 2018;29(7):990-997. ISSN 15408167.

[8] Potse M, Vinet A, Opthof T, Coronel R. Validation of a simple model for the morphology of the $\mathrm{T}$ wave in unipolar electrograms. American Journal of Physiology Heart and Circulatory Physiology 2009;297(2):H792-H801. ISSN 0363 6135.

[9] Ramirez J, Orini M, Tucker JD, Pueyo E, Laguna P. Variability of Ventricular Repolarization Dispersion Quantified by Time-Warping the Morphology of the T-Waves. IEEE Transactions on Biomedical Engineering jul 2017;64(7):16191630. ISSN 15582531.

\section{Address for correspondence:}

Name: Michele Orini

Address: University College London, Gower Street, London, UK.

E-mail address:m.orini@ucl.ac.uk 